\title{
Effect of Cefazolin Treatment on the Nonresonant Raman Signatures of the Metabolic State of Individual Escherichia coli Cells
}

\author{
Tobias J. Moritz, ${ }^{t, \ddagger}$ Douglas S. Taylor, ${ }^{\ddagger, \S}$ Christopher R. Polage," Denise M. Krol,, $\perp$ \\ Stephen M. Lane, ${ }^{*}$ and James W. Chan*,*,\#
}

\begin{abstract}
Biophysics Graduate Group, University of California, Davis, California 95616, NSF Center for Biophotonics Science and Technology, UC Davis, Sacramento, California 95817, Department of Pediatrics, UC Davis Medical Center, Department of Pathology and Laboratory Medicine, UC Davis Medical Center, Sacramento, California 95820, Department of Applied Science, University of California, Davis, California 95616, and Physics Division, Lawrence Livermore National Laboratory, Livermore, California 94550
\end{abstract}

Laser tweezers Raman spectroscopy (LTRS) was used to characterize the Raman fingerprints of the metabolic states of Escherichia coli ( $E$. coli) cells and to determine the spectral changes associated with cellular response to the antibiotic Cefazolin. The Raman spectra of $E$. coli cells sampled at different time points in the bacterial growth curve exhibited several spectral features that enabled direct identification of the growth phase of the bacteria. Four groups of Raman peaks were identified based on similarities in the time-dependent behavior of their intensities over the course of the growth curve. These groupings were also consistent with the different biochemical species represented by the Raman peaks. Raman peaks associated with DNA and RNA displayed a decrease in intensity over time, while protein-specific Raman vibrations increased at different rates. The adenine ring-breathing mode at 729 and the $1245 \mathrm{~cm}^{-1}$ vibration peaked in intensity within the first $10 \mathrm{~h}$ and decreased afterward. Application of principal component analysis (PCA) to the Raman spectra enabled accurate identification of the different metabolic states of the bacterial cells. The Raman spectra of cells exposed to Cefazolin at the end of log phase exhibited a different behavior. The 729 and 1245 $\mathrm{cm}^{-1}$ Raman peaks showed a slight decrease in intensity from 4 to $10 \mathrm{~h}$ after inoculation. Moreover, a shift in the spectral position of the adenine ringbreathing mode from 724 to $729 \mathrm{~cm}^{-1}$, which was observed during normal bacterial growth, was inhibited during antibiotic drug treatment. These results suggest that potential Raman markers exist that can

\footnotetext{
* To whom correspondence should be addressed. Phone: 925-423-3565. Fax: 925-424-2778. E-mail: chan19@1lnl.gov.

† Biophysics Graduate Group, University of California.

NSF Center for Biophotonics Science and Technology.

$\S$ Department of Pediatrics, UC Davis Medical Center.

"Department of Pathology and Laboratory Medicine, UC Davis Medical Center.

${ }^{\perp}$ Department of Applied Science, University of California.

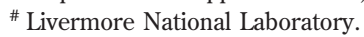

be used to identify $E$. coli cell response to antibiotic drug treatment.

Methods for early, rapid, and accurate identification of microbial pathogens and their drug resistant or susceptibility properties are very important in medicine to enable prompt implementation of therapy using the most effective targeted antibiotics. Infectionrelated mortality rates have been shown to be significantly lower in patient groups that receive immediate treatment. ${ }^{1,2}$ Current clinical methods that are used for pathogen identification and evaluation of their drug response have not significantly changed over the past few decades and mostly involve enrichment of a pure microbial colony and performing a series of tests for assessing either their morphological features or physiological characteristics based on specific metabolic reactions. The numerous tests and the culturing procedures can be time-consuming, leading to several days before a definitive diagnosis can be reached.

Raman spectroscopy has attracted much attention recently as a prospective new optical technique for rapid microbial detection and classification. ${ }^{3}$ Inelastic scattering of photons by intrinsic molecular bonds as a laser beam probes the microbial cells leads to the generation of a Raman spectral fingerprint that reflects their biochemical composition, which can be used as unique molecular signatures for microbial identification. As such, the technique can potentially be more accurate and sensitive than existing methods. The overall simplicity of the technique is also attractive, eliminating the need for extensive sample preparation and handling or the use of labels or stains. The ability to identify and classify clinically relevant microorganisms with high accuracy using Raman spectroscopy has been demonstrated. ${ }^{4-9}$ Moreover, studies have shown that Raman spectroscopy can be used for realtime monitoring of bacterial metabolism and growth. For example, studies on the growth of yeast cells ${ }^{10,11}$ and the molecular reactions in Escherichia coli cells ${ }^{12,13}$ have also been performed. To a lesser extent, Raman spectroscopy has been used to study

(1) Kollef, M. H. Clin. Infect. Dis. 2000, 31, S131-S138.

(2) Ibrahim, E. H.; Sherman, G.; Ward, S.; Fraser, V. J.; Kollef, M. H. Chest 2000, 118, 146-155.

(3) Harz, A.; Rosch, P.; Popp, J. Cytometry, Part A 2009, 75A, 104-113. 
the response of bacterial cells following exposure to antibiotic drugs. These studies have been performed primarily on bulk cell populations dried on a substrate ${ }^{14-16}$ and/or have specifically used UV resonance Raman spectroscopy ${ }^{14,15}$ to study changes in specific molecular vibrations such as the aromatic ring structures and bases of amino acids and DNA, whose signals are enhanced by the UV resonance process.

To our knowledge, a single-cell Raman study of the growth of normal and antibiotic exposed bacterial cells has not been reported. The capability to spectroscopically characterize single cells is essential for the development of clinical diagnostic tools that allow rapid detection of small subpopulations within a heterogeneous cell population. This point is elaborated upon in greater detail later in this article. Here, we present a comprehensive study utilizing laser tweezers combined with nonresonant, spontaneous Raman spectroscopy, ${ }^{17-20}$ a technique that enables global analysis of the molecular vibrations of individual, viable cells in solution, to compare the development of bacterial cells under normal growth conditions and when exposed to antibiotic drugs. Using $E$. coli bacterial cells as a model system, a detailed characterization of their Raman spectra during normal growth is first performed, with the goal of understanding the natural metabolic variability that is reflected in the Raman spectra of the cells. This information is critical in order to accurately assess the significance of spectral changes induced by environmental perturbations such as the introduction of antibiotic drugs. The Raman spectra of $E$. coli cells exposed to antibiotic treatment are next characterized and correlated to the normal growth spectra to identify the specific spectral markers associated with cellular drug response. This study demonstrates the potential of Raman spectroscopy as a powerful clinical and basic research tool to provide

(4) Xie, C.; Mace, J.; Dinno, M. A.; Li, Y. Q.; Tang, W.; Newton, R. J.; Gemperline, P. J. Anal. Chem. 2005, 77, 4390-4397.

(5) Buijtels, P.; Willemse-Erix, H. F. M.; Petit, P. L. C.; Endtz, H. P.; Puppels, G. J.; Verbrugh, H. A.; van Belkum, A.; van Soolingen, D.; Maquelin, K. J. Clin. Microbiol. 2008, 46, 961-965.

(6) Huang, W. E.; Griffiths, R. I.; Thompson, I. P.; Bailey, M. J.; Whiteley, A. S. Anal. Chem. 2004, 76, 4452-4458.

(7) Maquelin, K.; Choo-Smith, L. P.; Endtz, H. P.; Bruining, H. A.; Puppels, G. J. J. Clin. Microbiol. 2002, 40, 594-600.

(8) Maquelin, K.; Kirschner, C.; Choo-Smith, L. P.; Ngo-Thi, N. A.; van Vreeswijk, T.; Stammler, M.; Endtz, H. P.; Bruining, H. A.; Naumann, D.; Puppels, G. J. J. Clin. Microbiol. 2003, 41, 324-329.

(9) Buijtels, P. C. A. M.; Willemse-Erix, H. F. M.; Petit, P. L. C.; Endtz, H. P.; Puppels, G. J.; Verbrugh, H. A.; van Belkum, A.; van Soolingen, D.; Maquelin, K. J. Clin. Microbiol. 2008, 46, 961-965.

(10) Singh, G. P.; Volpe, G.; Creely, C. M.; Grotsch, H.; Geli, I. M.; Petrov, D. J. Raman Spectrosc. 2006, 37, 858-864.

(11) Huang, Y. S.; Karashima, T.; Yamamoto, M.; Hamaguchi, H. Biochemistry 2005, 44, 10009-10019.

(12) Xie, C. G.; Nguyen, N.; Zhu, Y.; Li, Y. Q. Anal. Chem. 2007, 79, 92699275.

(13) Chan, J. W.; Winhold, H.; Corzett, M. H.; Ulloa, J. M.; Cosman, M.; Balhorn, R.; Huser, T. Cytometry, Part A 2007, 71A, 468-474.

(14) Lopez-Diez, E. C.; Winder, C. L.; Ashton, L.; Currie, F.; Goodacre, R. Anal. Chem. 2005, 77, 2901-2906.

(15) Neugebauer, U.; Schmid, U.; Baumann, K.; Holzgrabe, U.; Ziebuhr, W.; Kozitskaya, S.; Kiefer, W.; Schmitt, M.; Popp, J. Biopolymers 2006, 82, 306-311.

(16) Escoriza, M. F.; Vanbriesen, J. M.; Stewart, S.; Maier, J. Appl. Spectrosc. 2006, 60, 971-976.

(17) Xie, C. G.; Dinno, M. A.; Li, Y. Q. Opt. Lett. 2002, 27, 249-251.

(18) Chan, J.; Fore, S.; Wachsman-Hogiu, S.; Huser, T. Laser Photonics Rev. 2008, 2, 325-349.

(19) Chan, J. W.; Esposito, A. P.; Talley, C. E.; Hollars, C. W.; Lane, S. M.; Huser, T. Anal. Chem. 2004, 76, 599-603.

(20) Creely, C. M.; Singh, G. P.; Petrov, D. Opt. Commun. 2005, 245, 465-470. direct information of the metabolic state of a cell and determine its drug resistance or susceptibility. Our work addresses the demand for a clinical diagnostic method that will decrease evaluation time and increase sensitivity, which ultimately will lead to decreased medical care costs. ${ }^{21}$

\section{EXPERIMENTAL SECTION}

Growth, Culturing, and Antibiotic Treatment of E. coli Cells. E. coli (ATCC 25922) cultures were started on a blood agar plate (Remel) approximately $12 \mathrm{~h}$ prior to the experiments. A single $E$. coli colony was then inoculated from the plate into 20 $\mathrm{mL}$ of $\mathrm{L}$ broth (Gibco) in a conical tube (BD Falcon 352070) and incubated at $T=37^{\circ} \mathrm{C}, f=225 \mathrm{rpm}$ in a shaker incubator (Excella E25). The E. coli growth curve was recorded by sampling the bacterial culture in $1-2 \mathrm{~h}$ time steps. One milliliter of sample was transferred into a cuvette (Fisherbrand), and the absorbance at $\lambda$ $=600 \mathrm{~nm}$ was measured with a photospectrometer (Shimadzu, Biospec 1601). The absorbance values were plotted as a function of time on a semilogarithmic scale. The antibiotic experiments were performed by exposing the bacterial cultures to the antibiotic drug Cefazolin at a concentration of $100 \mu \mathrm{g} / \mathrm{mL} 3.5 \mathrm{~h}$ after inoculation, which coincided with the end of $\log$ phase. A total of seven broth cultures using three different batches of Luria-Bertani broth were independently grown for spectral analysis, five without antibiotics and two with Cefazolin treatment.

Laser Tweezers Raman Spectroscopy (LTRS) Setup. The LTRS system used in this study has been described in detail previously. ${ }^{22}$ A single laser beam from a $785 \mathrm{~nm} \mathrm{CW}$ diode laser $\left(P_{\text {output }}=80 \mathrm{~mW}\right.$, CrystaLaser $)$ coupled into an inverted microscope (Olympus IX71) was used to trap individual cells and generate Raman spectra. An optical isolator (THORLABS, IO-5-780-HP) placed in front of the laser beam was used to stabilize the laser output power. A 10x Kepler telescope expanded the beam to overfill the back aperture of the microscope objective (Olympus 60×/1.2 NA water immersion) in order to generate a tightly focused laser beam for stable optical trapping of the bacteria in solution. A laser spot of about $1 \mu \mathrm{m}$ diameter and $28 \mathrm{~mW}$ at the sample was achieved. The Raman signals from the probed cells were detected by a fibercoupled spectrograph (Acton, SpectraPro 2300i) equipped with a 600 grooves/mm grating and a thermoelectrically cooled CCD camera (PI/Acton, PIXIS 100).

Raman Data Acquisition and Processing. An amount of $100 \mu \mathrm{L}$ of bacterial solution taken directly from the $\mathrm{LB}$ was added to $1 \mathrm{~mL}$ of phosphate-buffered saline (PBS) solution and pipetted into a cell chamber (Molecular Probes, Attofluor) with a fusedsilica microscope coverslip. Due to the high concentration of bacteria in this solution and the time it took to acquire the Raman spectra ( $\sim 60 \mathrm{~s})$, on average five bacterial cells were optically trapped simultaneously within the laser's focal volume for every spectral acquisition.

The commercially available software MATLAB was used for all data processing. A background spectrum of the solvent (100 $\mu \mathrm{L}$ LB in $1 \mathrm{~mL}$ of PBS) was subtracted from every Raman

(21) Doern, G. V.; Vautour, R.; Gaudet, M.; Levy, B. J. Clin. Microbiol. 1994, 32, 1757-1762.

(22) Moritz, T. J.; Brunberg, J. A.; Krol, D. M.; Wachsmann-Hogiu, S.; Lane, S. M.; Chan, J. W. J. Raman Spectrosc. 2009, 41, 33-39. 
spectrum. Spectra were cropped to a spectral region of interest ranging from 600 to $1779 \mathrm{~cm}^{-1}$, which covers the fingerprint region of most biological materials. A baseline correction algorithm that uses iterative polynomial fitting was applied to each spectrum. ${ }^{23}$ The intensities of the spectra were normalized to the total area under the curve, and the Savitzky-Golay filter ( $\operatorname{span}=7$, polynomial degree $=2$, curve fitting toolbox in MATLAB) was used to reduce the noise of the spectra.

Time traces of the evolution of specific Raman peaks during bacterial growth were generated by plotting the intensity values for specific peaks as a function of time. Intensity values were obtained by adding the maximum intensity and the intensity values of the two neighboring channels for each Raman peak. The peak intensities in these time traces were normalized to the peak intensities at $4 \mathrm{~h}$ after inoculation of the bacteria culture to provide a measure of relative intensity changes.

Principal component analysis (PCA) was used to separate and group the time-dependent Raman spectra. ${ }^{24}$ For this analysis, the Raman spectra were further cropped to the spectral region from 710 to $1280 \mathrm{~cm}^{-1}$ to take into account only the spectral components with the largest, time-dependent absolute intensity changes.

Standard deviation calculations for the difference Raman spectra $\left(\delta_{\mathrm{B}-\mathrm{A}}\right)$ were obtained from the standard deviations of the individual spectra $\left(\delta_{\mathrm{A}}, \delta_{\mathrm{B}}\right)$ using error propagation rules:

$$
\delta_{\mathrm{B}-\mathrm{A}}=\sqrt{\left(\delta_{\mathrm{B}}\right)^{2}+\left(\delta_{\mathrm{A}}\right)^{2}}
$$

\section{RESULTS AND DISCUSSION}

Correlation of Raman Spectroscopic Changes to Metabolic States of $\boldsymbol{E}$. coli. Figure $1 \mathrm{~A}$ shows a normal $E$. coli growth curve displaying log and stationary growth phases. Cell samples were obtained for Raman analysis at different time points as indicated on the growth curve (a, b, c). Raman spectra of $E$. coli cells taken during (a) log phase, (b) growth at the end of log phase, and (c) stationary phase are shown in Figure 1B. Each spectrum is an average of 75 bacterial cells. The three Raman spectra representing different metabolic states display significant changes in Raman peak intensities and peak ratios. An analysis of the time-dependent changes in the individual Raman peaks is shown in the peak intensity time traces in Figure 2. Four groups of Raman peaks can be identified based on similarities in their time trace profiles. Moreover, the peaks also group according to the same type of biomolecules to which they are assigned, as shown in Table 1. Raman frequencies in groups A and B are specific to DNA, and groups $\mathrm{C}$ and $\mathrm{D}$ are associated with proteins. In general, most of the DNA peaks exhibit a continuous decrease in intensity, while most protein peaks increase in intensity. However, several peaks exhibit a different trend. The 729 and $1245 \mathrm{~cm}^{-1}$ peaks in group $\mathrm{B}$, which are related to adenine, increase in intensity for the first $10 \mathrm{~h}$ followed by a decrease, while several protein peaks (group D) show a minimal increase. The most significant change in peak intensity can be observed for the 784 and 811 $\mathrm{cm}^{-1}$ Raman frequencies related to DNA/RNA. The peak intensity is reduced by a factor of 2 during transition from $\log$ phase to the beginning of stationary phase (points a to $\mathrm{b}$ in

(23) Lieber, C. A.; Mahadevan-Jansen, A. Appl. Spectrosc. 2003, 57, 1363-1367. (24) Ringner, M. Nat. Biotechnol. 2008, 26, 303-304.

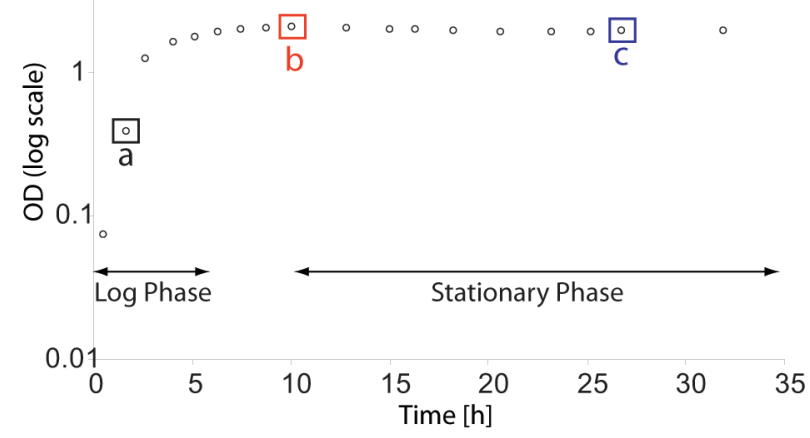

B

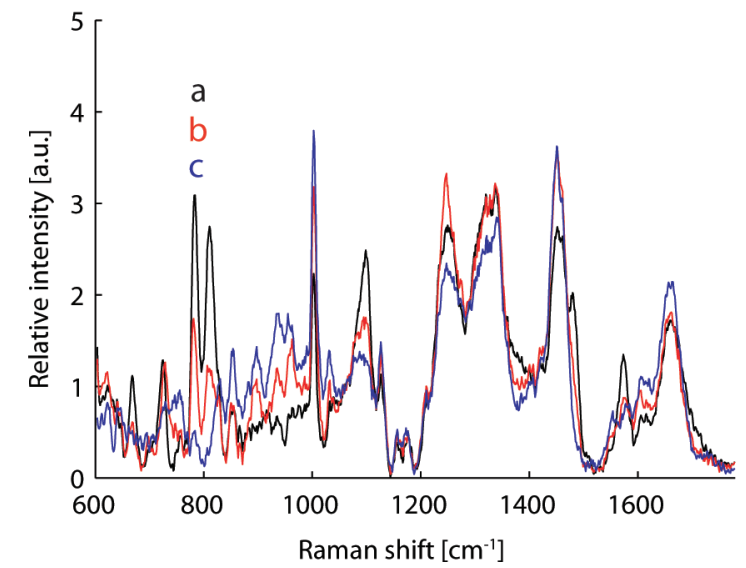

Figure 1. Raman spectra of $E$. coli cells taken at different time points on the growth curve. (A) A typical E. coli growth curve. Cells at (a) log phase, (b) end of log phase/beginning of stationary phase, and (c) stationary phase were analyzed by Raman spectroscopy. (B) Mean Raman spectra of cells at time points a-c.

\section{Table 1. Raman Frequencies and Their Peak Assignments Grouped by Their Time-Dependent Trace Profiles Shown in Figure $2^{a}$}

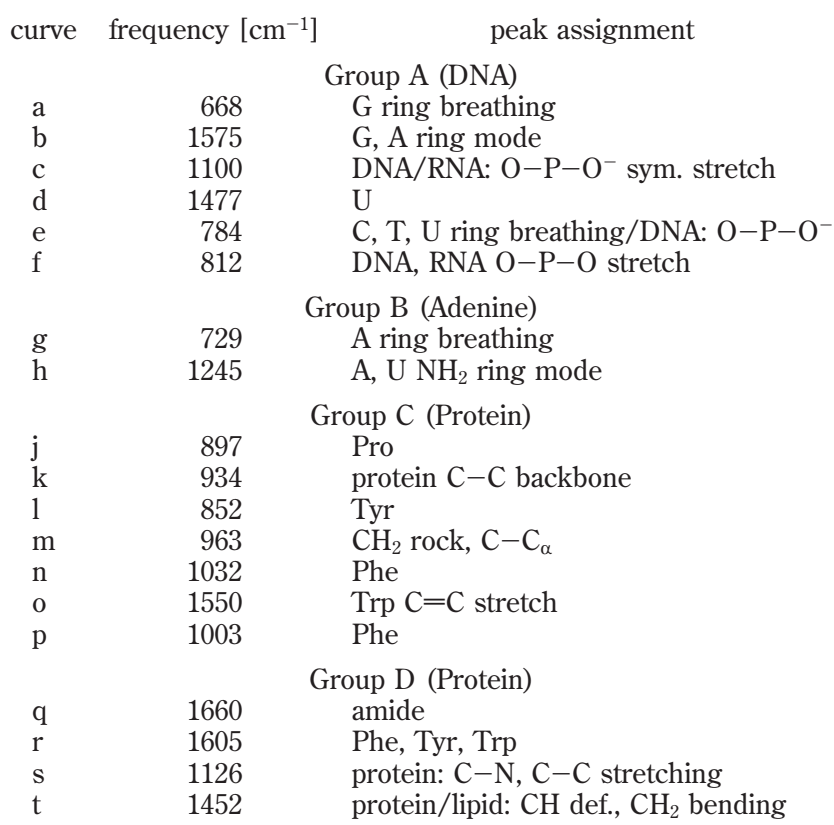

${ }^{a}$ Refs 4, 6, 7, 9, 15, 16, 28-31, 33, 34. 
A

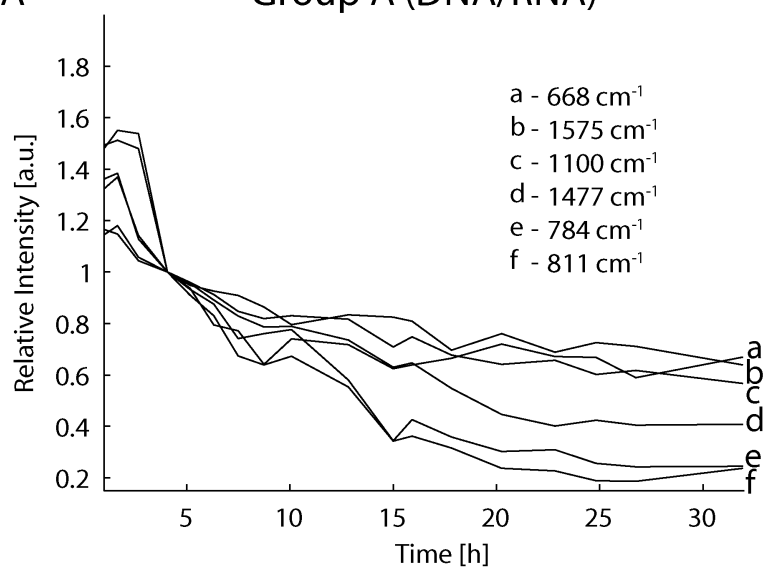

B

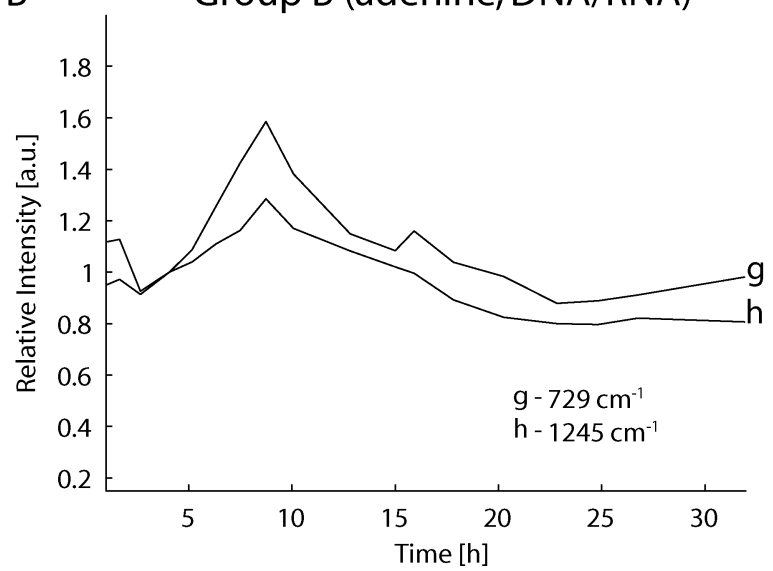

C

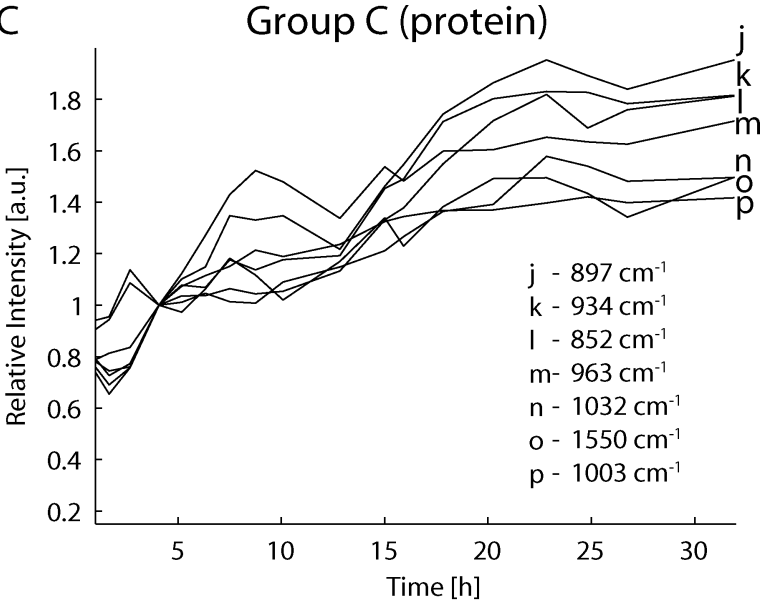

D

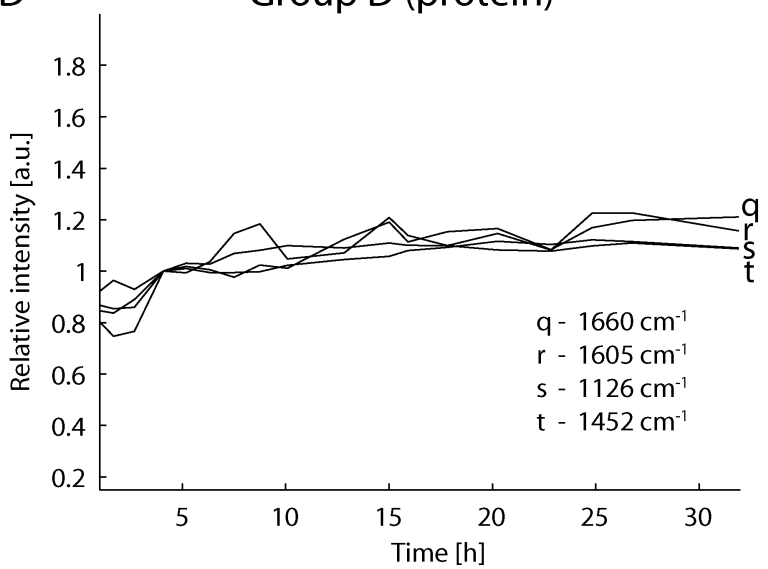

Figure 2. Time traces of relative Raman peak intensities during normal cell growth. Peak traces were grouped based on similar profiles. Grouping of the Raman frequencies also coincided with the molecular species assigned to each of the peaks. (A and B) Two groups that are specific to DNA/RNA but exhibit different profiles. (C and D) Two groups that are specific to protein but exhibit different profiles. Individual traces are labeled a through $t$, and their corresponding Raman frequencies are shown.

Figure 1A), which can be associated with cell division. Bacterial cells double the amount of DNA just before binary fission occurs but have only one DNA complement during stationary phase. ${ }^{25}$ The decrease in DNA/RNA-specific peak intensities and the increase in protein-specific peak intensities over time indicate an increase in protein synthesis as a response to environmental stress induced by the depletion of nutrients. This is a known stress response for bacteria. ${ }^{25-27}$ The Raman spectra do not change significantly during stationary phase, which is reflected in the constant peak intensities after approximately $25 \mathrm{~h}$ as shown in Figure 2. During stationary phase, no DNA or protein synthesis occurs as the metabolism of bacterial cells becomes inactive. ${ }^{25,26}$ It is important to emphasize that, even though no changes in the optical density (OD) can be observed in the growth curve (Figure 1A) after approximately $10 \mathrm{~h}$, Raman spectroscopy is sensitive enough to detect changes in the bacterial cells up to $25 \mathrm{~h}$. This is perhaps not surprising considering that OD measurements are only sensitive to the concentration of cells in solution but not to physiological changes of the cells themselves, whereas Raman

(25) Schaechter, M.; Ingraham, J. L.; Neidhard, F. C. Microbe; ASM Press: Washington, DC, 2006.

(26) Madigan, M.; Martinko, J. M.; Dunlap, P. V.; Clark, D. P. Brock Biology of Microorganisms, 12th ed., Pearson Benjamin Cummings: San Francisco, CA, 2009.

(27) Chung, H. J.; Bang, W.; Drake, M. A. Compr. Rev. Food Sci. Food Saf. 2006, $5,52-64$. spectroscopy can provide specific markers of DNA, RNA, and protein, whose ratios in a bacterial cell can be highly dependent on its metabolic state.

Bacterial cells in different metabolic states can be even more clearly identified and visualized by performing PCA on the Raman spectra. The scatter plot of the first (PC1) and second (PC2) principal components (Figure 3A) shows several groups of cells that are clearly separated according to their metabolic states. Three groups can be identified based on the time points on the growth curve, $0.5-2.5 \mathrm{~h}$ corresponding to exponential growth (log phase), 4-16 h corresponding to growth at the end of log phase, and $18-32 \mathrm{~h}$ corresponding to stationary phase. Furthermore, groups of cells at different time points within the $12 \mathrm{~h}$ time span during the end of $\log$ phase and the $5 \mathrm{~h}$ time span during

(28) Ruiz-Chica, A. J.; Medina, M. A.; Sanchez-Jimenez, F.; Ramirez, F. J. J. Raman Spectrosc. 2004, 35, 93-100.

(29) Benevides, J. M.; Tsuboi, M.; Bamford, J. K. H.; Thomas, G. J. Biophys. J. 1997, 72, 2748-2762.

(30) Ruiz-Chica, J.; Medina, M. A.; Sanchez-Jimenez, F.; Ramirez, F. J. Biochim. Biophys. Acta 2003, 1628, 11-21.

(31) Puppels, G. J.; Otto, C.; Greve, J.; Robertnicoud, M.; Arndtjovin, D. J.; Jovin, T. M. Biochemistry 1994, 33, 3386-3395.

(32) Hayashi, H.; Nishimura, Y.; Tsuboi, M.; Sekimizu, K.; Nakanishi, Y.; Natori, S. Biopolymers 1985, 24, 1107-1111.

(33) Thomas, G. J.; Benevides, J. M.; Overman, S. A.; Ueda, T.; Ushizawa, K.; Saitoh, M.; Tsuboi, M. Biophys. J. 1995, 68, 1073-1088.

(34) Serban, D.; Benevides, J. M.; Thomas, G. J. Biochemistry 2002, 41, 847853. 
A

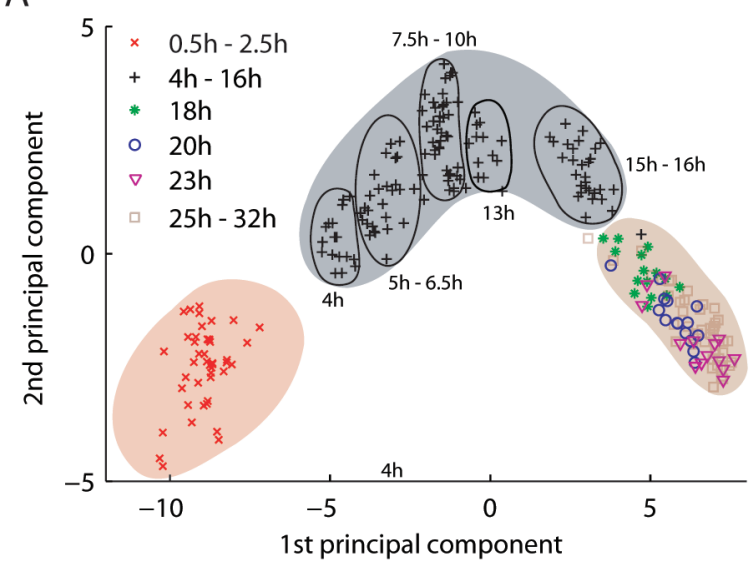

B

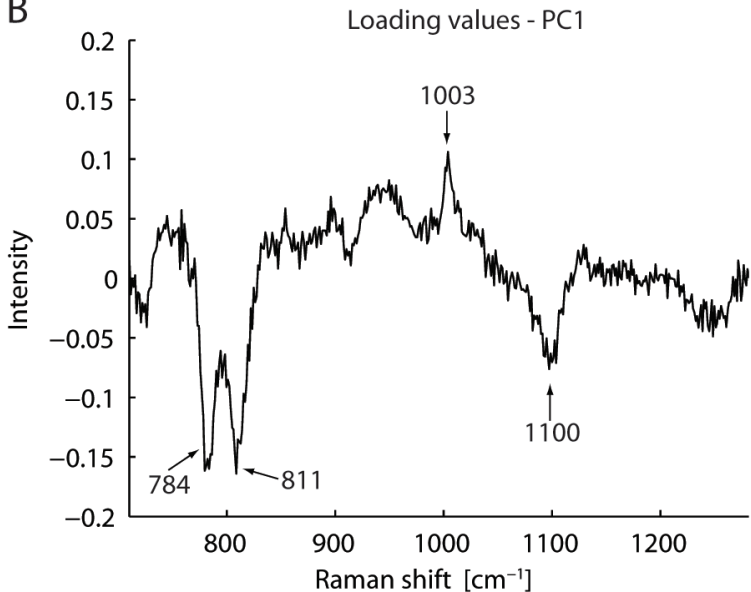

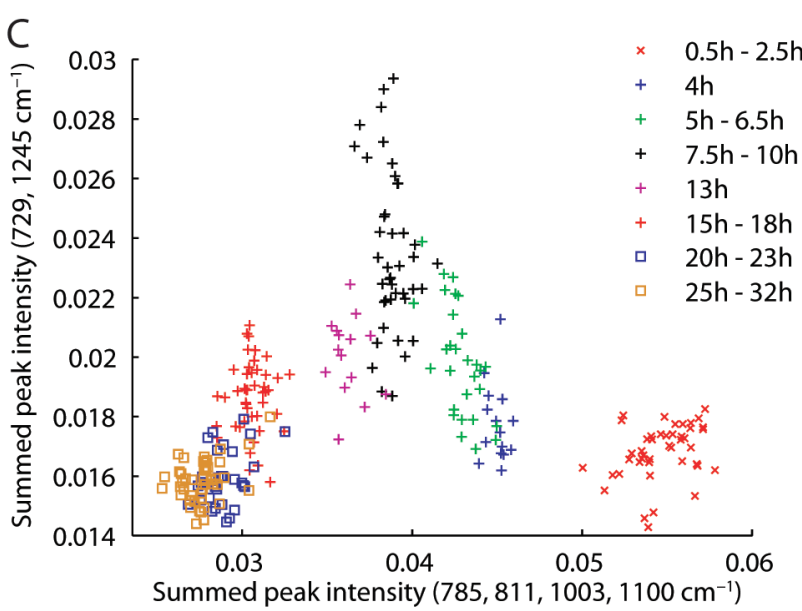

D

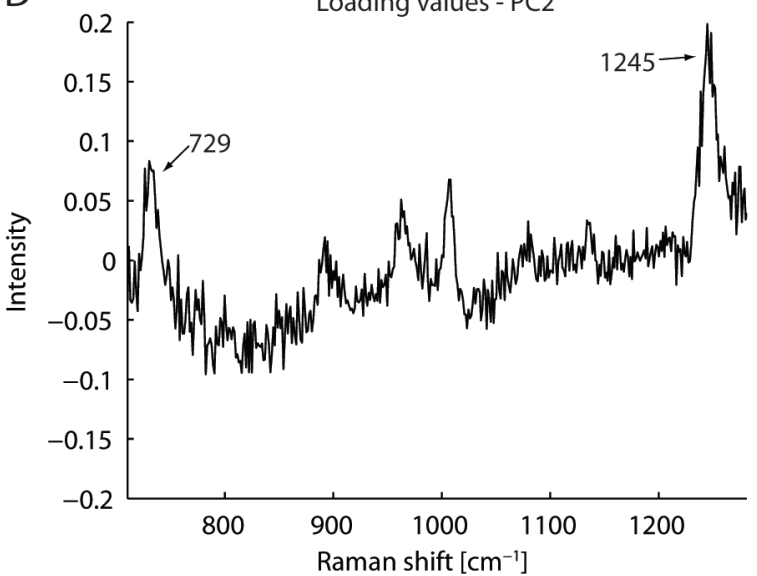

Figure 3. Principal component analysis (PCA) of Raman spectra for normal E. coli growth. (A) PCA scatter plot using the PC1 and PC2 values. Raman spectra taken at $0.5-2.5,4-16,18,20,23$, and $25-32 \mathrm{~h}$ are represented by different symbols (see figure legend). Shaded regions overlaid on the data points serve only as visual guides to the eye. The $4-16 \mathrm{~h}$ time frame is further separated into shorter time frames (black circles). Different groupings of Raman spectra taken at different time points during normal cell growth indicate the ability to identify metabolic states of a cell by its Raman spectra. (B and D) Loading value plots for PC1 and PC2, respectively. The primary Raman peaks that contribute to the data separation in the PCA plots are labeled. (C) Scatter plot of the sum of the intensities of relevant Raman peaks identified by the PCs. Peak intensities are calculated using five channels for each peak.

stationary phase $(18-23 \mathrm{~h})$ can be discriminated as well. Separation of these groups is better at the end of log phase (earlier time) than during stationary phase (later time) based on the number of outliers for the 20 and $23 \mathrm{~h}$ groups (Figure $3 \mathrm{~A}$ ). Bacterial cells at time points later than $25 \mathrm{~h}$ are indistinguishable from and overlap with the bacterial cells within the $18-23 \mathrm{~h}$ time points.

The improved group separation within the $4-16 \mathrm{~h}$ time points compared to the $18-32 \mathrm{~h}$ time points suggests that changes in cell physiology are much larger during late exponential/early stationary phase (up to $16 \mathrm{~h}$ of growth) than during stationary phase. The poor group separation for the latter stages of cell growth (greater than $18 \mathrm{~h}$ ) indicates that there is an increasingly heterogeneous population of cells at different growth stages, with some cells still actively undergoing metabolic changes while others are at rest. This would explain why some of the cells in the 20,23 , and $25-32 \mathrm{~h}$ groups are colocalized with the cell groups at earlier time points.

PCA of the Raman data confirms the detection of metabolic changes past $10 \mathrm{~h}$ of growth, which is not observable with simple OD measurements. These results highlight the importance of single-cell analysis over bulk cell analysis for detecting and evaluating cell growth.
The loading values of $\mathrm{PC} 1$ and $\mathrm{PC} 2$ plotted in Figure 3, parts $\mathrm{B}$ and $\mathrm{D}$, respectively, indicate the Raman peaks with the highest absolute variance over time and that contribute most to the separation observed in the PCA plot. PC1 and PC2 account for over $80 \%$ of the variance in the data. The peaks $784,811,1003$, and $1100 \mathrm{~cm}^{-1}$ were identified for PC1 and 729 and $1245 \mathrm{~cm}^{-1}$ for PC2. Plotting the sums of these peak intensity values for each Raman spectrum generates a scatter plot (Figure 3C) that displays the same cell group profile as the PCA plot, which confirms the most significant spectral peaks that change during the bacterial growth. This also suggests that the summed values of these peaks can be used as simple parameters to classify the metabolic state of an unknown $E$. coli bacterial cell after Raman analysis.

Escoriza et al. ${ }^{16}$ previously reported Raman changes associated with the different metabolic states in the normal growth of $E$. coli cells. They reported changes in Raman peaks corresponding to nucleic acid bases present in DNA/RNA molecules at 720, 785, and $1575 \mathrm{~cm}^{-1}$, which were consistent with the results reported in this study. Our single-cell Raman study is a comprehensive analysis that provides more detailed spectral and temporal information of all Raman spectral components, including 
A

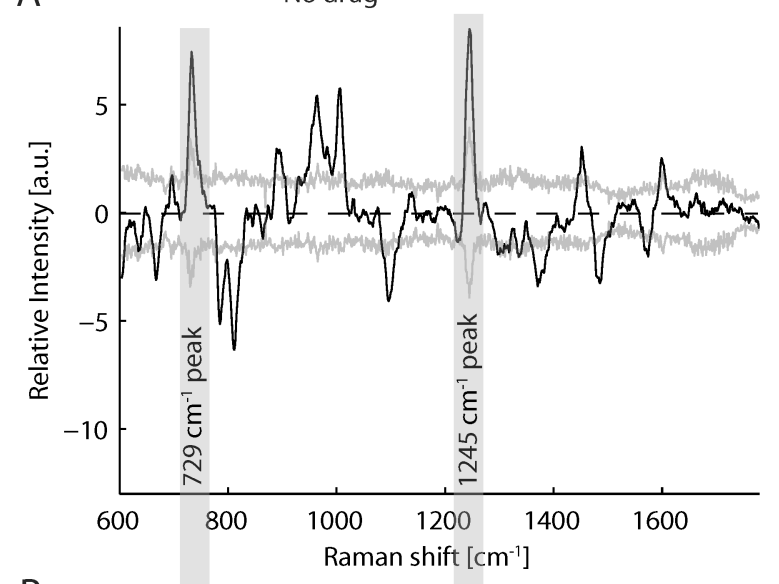

B

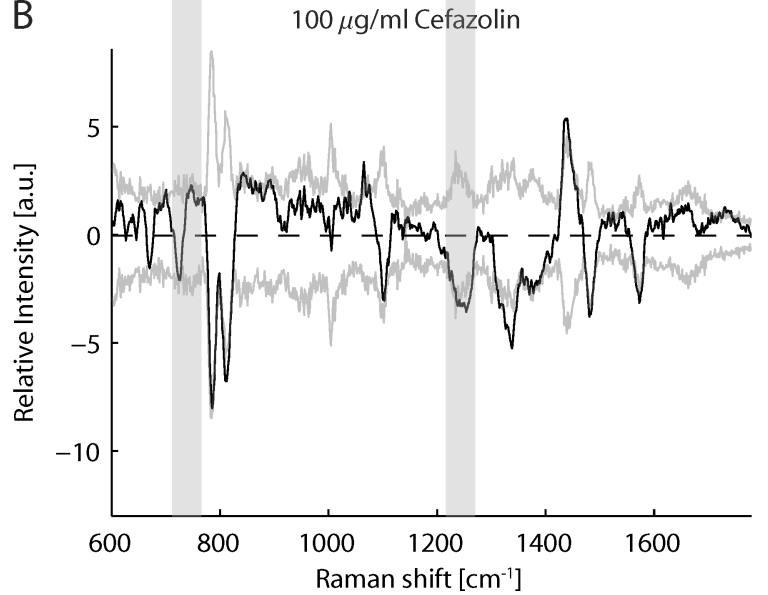

C

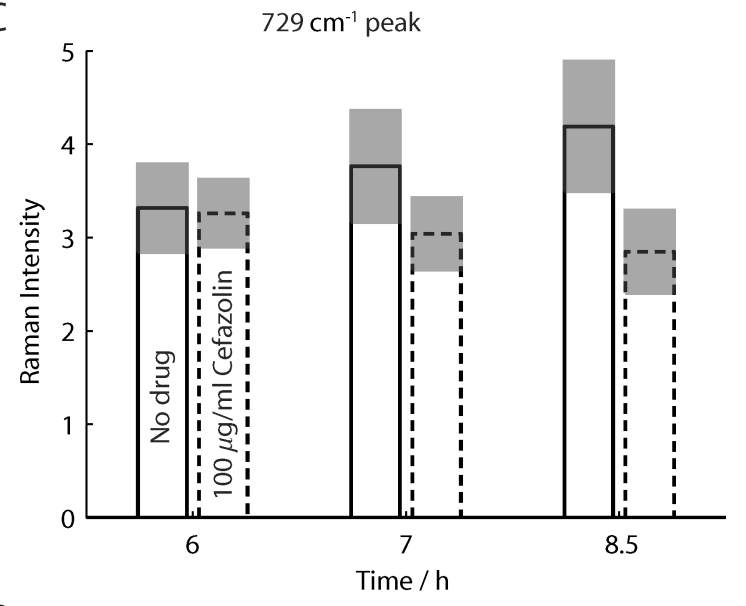

D

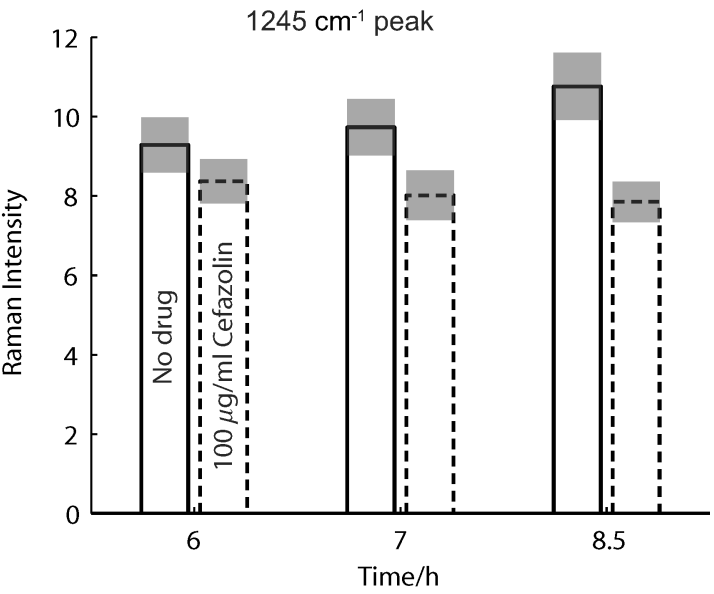

Figure 4. Raman spectral changes after antibiotic treatment. (A and B) Difference Raman spectra between 10 and $4 \mathrm{~h}$ after inoculation for normal growth and antibiotic treatment, respectively. Standard deviations are given as gray curves. Relevant differences between normal growth and antibiotic treatment conditions are visible in the 729 and $1245 \mathrm{~cm}^{-1}$ Raman peaks. (C and D) Time dependence of absolute Raman peak intensities for 729 and $1245 \mathrm{~cm}^{-1}$, respectively, comparing normal growth (solid bars) to drug treatment (dotted bars). Standard deviations are marked in gray.

protein-related peaks that change during cell growth. This detailed analysis is important in order to define a benchmark for the variability in the Raman spectra due to normal cell growth in order to evaluate and identify the significant spectral changes induced by abnormal growth conditions such as the exposure to antibiotic drugs, which is presented in the next section.

Raman Spectroscopic Changes after Antibiotic Treatment. Figure 4, parts A and B, shows the difference Raman spectra of E. coli cells undergoing normal growth and antibiotic treatment with $100 \mu \mathrm{g} / \mathrm{mL}$ Cefazolin, respectively. Difference spectra were calculated between the 10 and $4 \mathrm{~h}$ time points in order to visualize the maximum spectral changes over this time frame. Two distinct peaks at 729 and $1245 \mathrm{~cm}^{-1}$, which are highlighted in gray in parts $\mathrm{A}$ and $\mathrm{B}$ of Figure 4, were observed to be noticeably different in the two difference spectra. Plots of the temporal evolution of the 729 and $1245 \mathrm{~cm}^{-1}$ peak intensities in Figures 4, parts C and D, respectively, clearly show a different behavior for normal growth and antibiotic treatment. The intensities of the 729 and $1245 \mathrm{~cm}^{-1}$ Raman peaks, which increase in the $4-10 \mathrm{~h}$ time frame for normal $E$. coli growth, did not increase during antibiotic treatment. A detailed analysis of the $729 \mathrm{~cm}^{-1}$ spectral region revealed that a shift in the Raman peak from 724 to 729 $\mathrm{cm}^{-1}$ was, in fact, occurring for cells during normal growth (Figure 5A), which was not observed during Cefazolin treatment
(Figure 5B). This Raman frequency is specific to the ringbreathing mode of adenine, and the frequency change can be observed approximately $8 \mathrm{~h}$ after inoculation of the culture. The change of the maximum peak value of the $724 \mathrm{~cm}^{-1}$ Raman frequency appears to be associated with peak broadening.

The Raman spectroscopic differences observed in Figures 4 and 5 between normal growth and growth with drug exposure suggest that the cells undergo a physiological change involving the adenine environment during normal growth that is inhibited during antibiotic treatment. Both drug treatment Raman markers can be linked to this purine. The $729 \mathrm{~cm}^{-1}$ Raman frequency is specific to the ring-breathing mode of adenine, and $1245 \mathrm{~cm}^{-1}$ can be assigned to $\mathrm{NH}_{2}$ ring modes of $\mathrm{A}-\mathrm{T}$ and $\mathrm{A}-\mathrm{U}$ base pairs. ${ }^{28,29}$ It is known that the $1245 \mathrm{~cm}^{-1}$ peak can also be assigned to the amide III protein vibration, but since the time trace profile of this Raman peak does not follow any of the protein-specific peaks but instead has a similar trend as the adenine $729 \mathrm{~cm}^{-1}$ peak during normal growth (Figure 2B), we have attributed the $1245 \mathrm{~cm}^{-1}$ intensity change to DNA/RNA.

The adenine ring-breathing mode frequency is sensitive to different changes in DNA environment. ${ }^{30-32}$ We believe that the Raman frequency shift from 724 to $729 \mathrm{~cm}^{-1}$ for normal cell growth may be due to changes in secondary DNA structure. These changes can involve nicked DNA and altered base stacking geometry, which can be associated with relaxed 
A

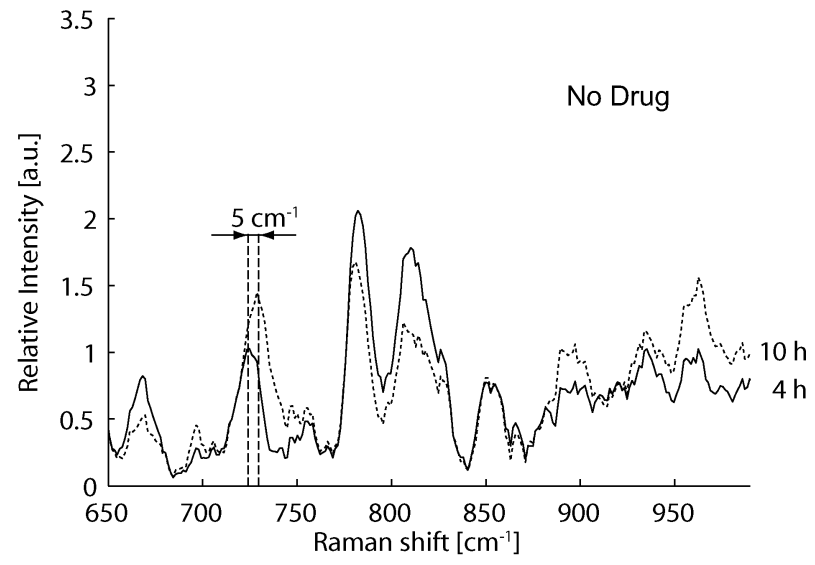

B

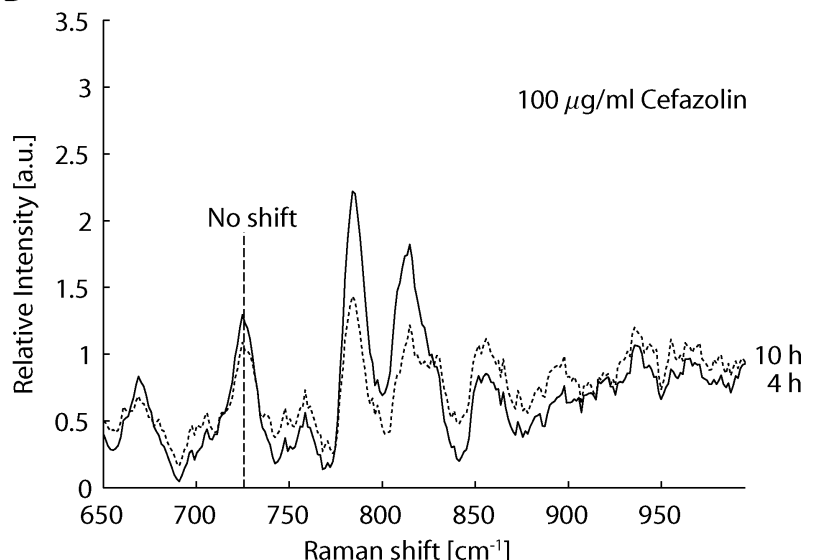

Figure 5. Analysis of the $729 \mathrm{~cm}^{-1}$ Raman peak for normal growth and antibiotic treatment. (A) E. coli Raman spectra 4 and $10 \mathrm{~h}$ after inoculation of the bacterial culture display a shift in position of the maximum intensity value of the adenine ring-breathing mode from 724 to $729 \mathrm{~cm}^{-1}$. (B) E. coli Raman spectra at 4 and $10 \mathrm{~h}$ during drug treatment display no spectral shift of the $724 \mathrm{~cm}^{-1}$ Raman peak.

supercoiling or even denaturing. ${ }^{31,32}$ Transitions to different DNA forms in E. coli have been reported, which leads to differences in the adenine ring-breathing mode ${ }^{33,34}$ Recently, nonenzymatic in vivo glycosylation (glycation) of chromosomal DNA in $E$. coli during normal growth was reported. ${ }^{35}$ During DNA glycation, sugar molecules bind to $\mathrm{NH}_{2}$ of adenine, which leads to alterations in DNA secondary structure and destabilization of base pairing, which can go as far as unpairing of the bases. ${ }^{36}$ DNA glycation is strongest during early stationary phase, ${ }^{35}$ which coincides with our spectroscopic data. The $729 \mathrm{~cm}^{-1}$ as well as the $1245 \mathrm{~cm}^{-1}$ Raman peak intensities were highest during that growth phase. The observed shift of the adenine ring-breathing mode could be correlated to the relaxation of secondary DNA structure, and the increase in $1245 \mathrm{~cm}^{-1}$ peak intensity may also be associated with unstacking of thymine rings. ${ }^{31}$ Our assignment of the intensity increase of the 1245 $\mathrm{cm}^{-1}$ Raman peak without a corresponding frequency shift to the unstacking of thymine rings related to adenine glycation

(35) Mironova, R.; Niwa, T.; Handzhiyski, Y.; Sredovska, A.; Ivanov, I. Mol. Microbiol. 2005, 55, 1801-1811.

(36) Sengupta, B.; Uematsu, T.; Jacobsson, P.; Swenson, J. J. Phys. Chem. B 2007, 111, 646-651. is based on the Raman hypochromism effect. ${ }^{37-40}$ Briefly, in this effect, conformational changes to a DNA molecule can result in changes in Raman band intensity without a change in its frequency. A conformational change from a less ordered to an ordered state (e.g., single strands to double-strand helix) decreases the intensity of some DNA Raman bands, which is consistent with our data (i.e., in our case, an increase in intensity is observed due to a more disordered state). Hypochromism relies on the interdependence of Raman scattering intensity and electronic absorption, which is reduced for stacked bases compared to unstacked bases. ${ }^{39}$ The knowledge that nonenzymatic glycosylation results in changes to the secondary DNA structure involving unstacking of $\mathrm{A} / \mathrm{T}$ base pairs and the good agreement between the observed Raman spectral changes and previously reported glycation effects both occurring during early stationary phase for normal $E$. coli growth leads us to suggest glycation as a possible explanation for the observed spectral changes. We acknowledge that alternative effects could also be responsible for the observed Raman spectral effects.

\section{CONCLUSIONS}

We have demonstrated the use of LTRS for the identification and discrimination of the metabolic states of individual bacterial cells with and without Cefazolin treatment. To our knowledge, this is the first reported observation of a spectral shift in the adenine-specific $724 \mathrm{~cm}^{-1}$ Raman peak associated with normal E.coli cell growth, which is inhibited upon Cefazolin treatment. Furthermore, evidence has been provided linking the $724 \mathrm{~cm}^{-1}$ band to the $1245 \mathrm{~cm}^{-1}$ Raman frequency, based on similarities in peak time traces and changes to both peaks during treatment. When compared to Raman spectroscopy of bulk, dried cell samples, LTRS provides new and unique capabilities for many different scientific and clinical applications. LTRS enables spectral analysis of individual live bacterial cells under near physiological conditions (LB culture media or saline solution) without extensive sample preparation in order to maintain cell viability and obtain results that better reflect the natural biological state of the cell. Therefore, this technique has the potential to improve the understanding of dynamic cellular mechanisms or responses at a single-cell level rather than averaged over large numbers of cells in diverse states. Single-cell Raman analysis may also facilitate the recognition of subpopulations with unique metabolic, phenotypic, or antimicrobial responses (resistance). This is potentially important clinically given that heterogeneous resistance to antibiotics has been reported for Gram-positives, Gram-negatives, and anaerobes, which is usually due to mutation and or differential expression within what is otherwise a clonal population. Genetically distinct strains present simultaneously have also been described. Infections with high initial numbers of bacteria or chronic, partially treated infections are the typical situations where this is a problem. The most significant potential of single-

(37) Duguid, J. G.; Bloomfield, V. A.; Benevides, J. M.; Thomas, G. J. Biophys. J. 1995, 69, 2623-2641.

(38) Painter, P. C.; Koenig, J. L. Biopolymers 1976, 15, 241-255.

(39) Duguid, J. G.; Bloomfield, V. A.; Benevides, J. M.; Thomas, G. J. Biophys. J. 1996, 71, 3350-3360.

(40) Boernstein, L. Nucleic Acids-Spectroscopic and Kinetic Data, Physical Data I; Springer Verlag: New York, Berlin, Heidelberg, 1990. 
cell Raman detection for clinical diagnostics is the ability to evaluate mixed bacteria populations directly from clinical specimens (via minimal processing and microfluidics). The ability to analyze single cells may also reduce the required time at which bacterial response can be observed or phenotypic/ metabolic state can be assessed since fewer cells are needed and no prolonged upfront growth is required. Moreover, singlecell detection is needed in any type of cell sorting scheme such as separating live and dead cells in a high-throughput fashion by taking advantage of the Raman spectroscopic markers for cell death. The work presented herein lays the foundation for establishing LTRS as a single-cell technique for assessing bacterial response to drug treatment. Future studies will focus on studying the response of different types of bacteria to a variety of antibiotic drugs in order to build up a database of Raman signatures for identifying bacteria cell types and drug efficacy. Such an extensive investigation can be used to evaluate the applicability of the observed spectroscopic effects in this study for other bacterial types and strains.

\section{ACKNOWLEDGMENT}

We thank Distinguished Professor John Roth (Department of Microbiology at University of California, Davis) for a very helpful discussion. This work has been supported by funding from the National Science Foundation. The Center for Biophotonics, an NSF Science and Technology Center, is managed by the University of California, Davis, under cooperative agreement no. PHY 0120999. D. Taylor acknowledges support from the Children's Miracle Network, University of California, Davis.

Received for review October 16, 2009. Accepted February 12, 2010.

AC902351A 